STRUCTURAL BIOLOGY COMMUNICATIONS

ISSN 2053-230X

Received 9 July 2015

Accepted 30 September 2015

Edited by N. Sträter, University of Leipzig, Germany

Keywords: fatty-acid synthase; fatty-acid synthesis; multienzyme; tuberculosis; mycolic acid.

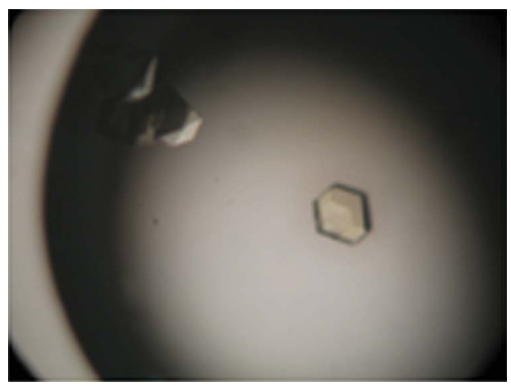

OPEN $\odot$ ACCESS

\section{Crystallization and X-ray diffraction studies of a complete bacterial fatty-acid synthase type I}

\author{
Mathias Enderle, ${ }^{\mathrm{a}, \mathrm{b}}$ Andrew McCarthy, ${ }^{\mathrm{c}}$ Karthik Shivaji Paithankar ${ }^{\mathrm{a} *}$ and Martin \\ Grininger $^{\mathrm{a}, \mathrm{b}}$ *
}

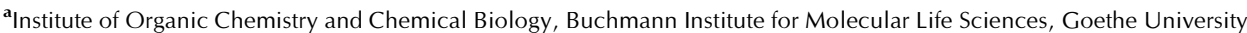
Frankfurt, Max-von-Laue-Strasse 15, 60438 Frankfurt am Main, Germany, ${ }^{\mathbf{b}}$ Department of Membrane Biochemistry, MaxPlanck-Institute of Biochemistry, Am Klopferspitz 18, 82152 Martinsried, Germany, and ${ }^{\mathrm{c}}$ EMBL Grenoble, 71 Avenue des Martyrs, 38042 Grenoble CEDEX 9, France. *Correspondence e-mail: paithankar@em.uni-frankfurt.de, grininger@chemie.uni-frankfurt.de
\end{abstract}

While a deep understanding of the fungal and mammalian multi-enzyme type I fatty-acid synthases (FAS I) has been achieved in recent years, the bacterial FAS I family, which is narrowly distributed within the Actinomycetales genera Mycobacterium, Corynebacterium and Nocardia, is still poorly understood. This is of particular relevance for two reasons: (i) although homologous to fungal FAS I, cryo-electron microscopic studies have shown that bacterial FAS I has unique structural and functional properties, and (ii) M. tuberculosis FAS I is a drug target for the therapeutic treatment of tuberculosis (TB) and therefore is of extraordinary importance as a drug target. Crystals of FAS I from C. efficiens, a homologue of $M$. tuberculosis FAS I, were produced and diffracted X-rays to about $4.5 \AA$ resolution.

\section{Introduction}

Fatty acids are important as principal components of cellular membranes, as post-translational modifiers of proteins, as a storage form of energy and as signalling molecules. The synthesis of fatty acids is performed in a catalytic cycle that is largely conserved in nature. Despite the conservation of the chemistry of synthesis, the structures of fatty-acid synthases (FAS) differ significantly in eukaryotes and bacteria. FAS are classified into type I and type II systems. Type I FAS are protein complexes of up to $2.7 \mathrm{MDa}$ in size. The catalytic sites are embedded in an elaborate architecture and substrates are shuttled as covalently bound molecules to acyl carrier protein (ACP) domains. The other 'conventional' FAS system that is present in most bacteria and mitochondria is referred to as type II, in which separate monofunctional proteins perform the specific steps required for fatty-acid synthesis (Schweizer \& Hofmann, 2004; Gago et al., 2011).

FAS type I multi-enzyme complexes have been extensively analyzed in recent years by X-ray crystallographic and cryoelectron microscopic (cryo-EM) studies (Maier et al., 2010; Grininger, 2014). Whereas the mammalian FAS I forms $\mathrm{X}$-shaped homodimeric complexes, the microbial (CMNbacterial and fungal) FAS I occurs in barrel-shaped multimeric complexes with $D_{3}$ symmetry. Microbial FAS I is found in essentially two stoichiometries: an $\alpha_{6} \beta_{6}$ heterododecameric complex occurring in most fungal FAS I and a homohexameric complex present in some fungi and in the $\mathrm{CMN}$-group bacteria. The structure of the $2.6 \mathrm{MDa}$ yeast FAS I has been determined by X-ray crystallography (Jenni et al., 2007; Lomakin et al., 2007; Johansson et al., 2008, 2009; Leibundgut et al., 2007). The $1.9 \mathrm{MDa}$ bacterial FAS I structure was 


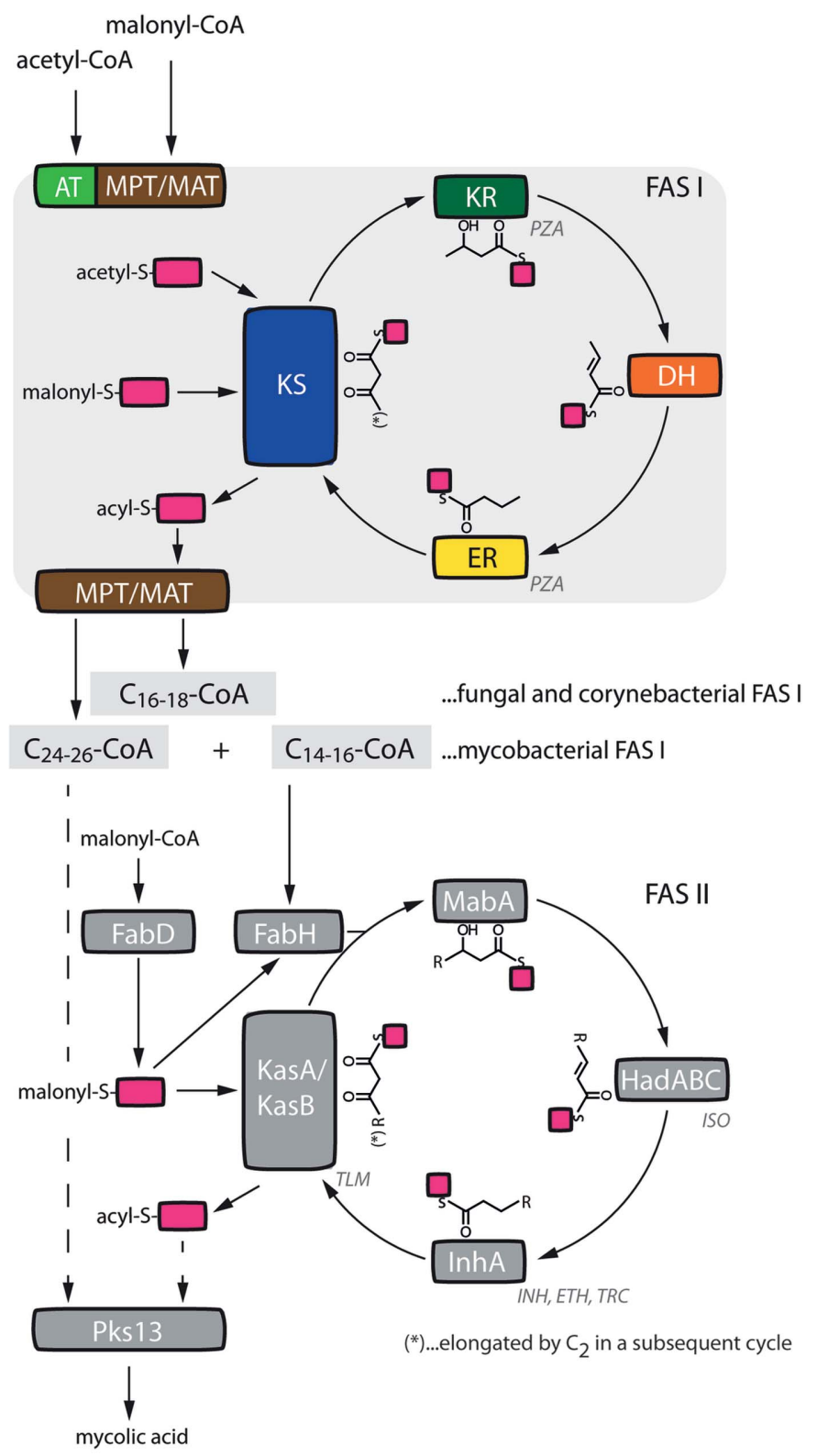

(a) characterized by cryo-EM, and a structural model was obtained by docking a fungal FAS I homology model (Boehringer et al., 2013). Cryo-EM studies of the conformational dynamics additionally contributed to the structural knowledge of FAS I systems (Brignole et al., 2009; Gipson et al., 2010; Ciccarelli et al., 2013), particularly by showing that bacterial and mammalian FAS I are conformationally dynamic, which critically determines their molecular mode (Grininger, 2014).

The bacterial FAS I system is only found in the CMN-group bacteria (Gago et al., 2011; Figs. $1 a$ and $1 b$ ). The most important representative of the FAS I-carrying bacteria is the highly pathogenic organism Mycobacterium tuberculosis, the causative agent of tuberculosis (TB). Functional studies have characterized $M$. tuberculosis FAS I as producing $\mathrm{C}_{16}$ and $\mathrm{C}_{26}$ fatty acids de novo, which serve as substrates for FAS type IImediated and Pks13-mediated synthesis of the branched mycolic acids, which can be up to $90 \mathrm{C}$ atoms in length (Kikuchi et al., 1992; Bhatt et al., 2007). The relevance of mycolic acids as an efficient barrier for M. tuberculosis is best illustrated by the use of three inhibitors of mycolic acid biosynthesis in antibiotic therapy for TB, pyrazinamide (PZA), isoniazid (INH) and ethambutol (Ma et al., 2010), with PZA identified as specifically targeting $M$. tuberculosis FAS I (Sayahi et al., 2011; Zimhony et al., 2000; Fig. 1a).

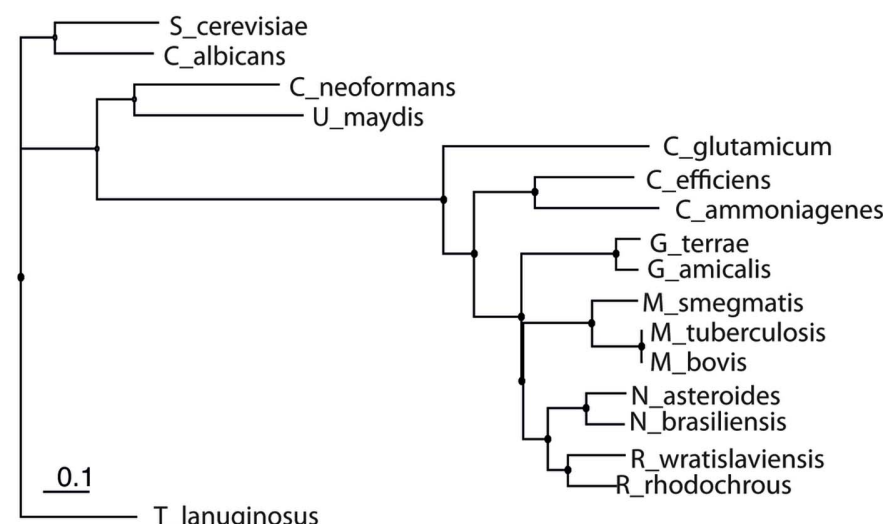

(b)

Figure 1

Overview of microbial de novo fatty-acid synthesis. (a) Synthetic machineries for fatty-acid production in bacteria and fungi. The grey background highlights the compartmentalized synthesis of acyl-CoA by the multifunctional FAS I systems. Key steps in the catalytic cycle of FAS I-mediated synthesis are as follows. The central ketoacyl synthase (KS) domain condenses ac(et)yl with malonyl to form $\beta$-ketobutyryl. Further, the compound is reduced by a ketoacyl reductase (KR) to $\beta$-hydroxyacyl, dehydrated by a dehydratase (DH) to produce enoyl, and again reduced by an enoyl reductase (ER). Substrate CoA esters are turned into ACP esters by transferases. ACPs (boxes in magenta) then shuttle substrates and intermediates to the active sites of the catalytic domains. Note that in FAS I, ACPs are part of the multienzyme. Also note that MPT (malonyl palmitoyl transferase) in fungal FAS I loads malonyl and unloads palmitoyl, and MAT (malonyl acyl transferase) in bacteria loads malonyl and unloads acyl chains of various chain lengths. The terminal thiol group of ACP is indicated to highlight that substrates and intermediates are provided as thioesters. Product specificities for corynebacterial, mycobacterial and fungal FAS I systems are given as reported in this study (C. efficiens FAS I) and previously: C. ammoniagenes FAS I (Stuible et al., 1997), M. tuberculosis and M. smegmatis FAS I (Kikuchi et al., 1992; Zimhony et al., 2004; Peterson \& Bloch, 1977) and Saccharomyces cerevisiae FAS I (Sumper et al., 1969; Kresze et al., 1977). Separate proteins of the FAS II are labelled as occurring in M. tuberculosis fatty-acid synthesis (Gago et al., 2011; Bhatt et al., 2007; Sacco et al., 2007). Pharmaceutically relevant inhibitors of $M$. tuberculosis fatty-acid synthesis are included in the scheme: pyrazinamide (PZA; Sayahi et al., 2011), isoxyl (ISO; Gannoun-Zaki et al., 2013), thiolactomycin (TLM; Kremer et al., 2000), isoniazid (INH), ethionamide (ETH) and triclosan (TRC) (Sullivan et al., 2006; Banerjee et al., 1994). (b) Phylogenetic analysis of selected bacterial and fungal FAS I. For the calculation of the phylogenetic tree, dual-chain fungal FAS I were submitted as $\beta / \alpha$-fused pseudo-single chains. For the analysis, FAS I with the following GenBank accession Nos. were used: Candida albicans (X74952.1, L29063.1), Saccharomyces cerevisiae (CAA82025.1, CAA97948.1), Cryptococcus neoformans (AAW43793.1, AAW43793.1), Ustilago maydis (XP_759118.1), Corynebacterium glutamicum (YP_225128), C. efficiens (NP_739002.1), C. ammoniagenes (CAA46024.1), Gordonia terrae (WP_004019558.1), G. amicalis (WP_024498183.1), Mycobacterium smegmatis (AAO43178.1), M. tuberculosis (NP_217040), M. bovis (NP_856198.1), Nocardia asteroides (WP_019045581.1), N. brasiliensis (GAJ86521.1), Rhodococcus wratislaviensis (GAF47416.1) and R. rhodochrous (ETT26757.1). Analysis was performed using the neighbour-joining algorithm with the Jones-Taylor-Thornton substitution model using the Ugene software package (Okonechnikov et al., 2012). 
We recently reported the cryo-EM structure of M. tuberculosis FAS I (Ciccarelli et al., 2013). For crystallographic studies, we identified Corynebacterium efficiens FAS I, which is $52 \%$ identical in sequence to $M$. tuberculosis FAS I, as a promising target. Here, we present the expression, purification, thermal stability data, crystallization and preliminary X-ray analysis of FAS I from C. efficiens.

\section{Materials and methods}

\subsection{Macromolecule production}

The cloning and purification of $M$. tuberculosis FAS I (UniProt accession code P95029) and C. ammoniagenes FAS I (UniProt accession code Q04846) have been described in detail elsewhere (Kikuchi et al., 1992); i.e. cloning with an infusion cloning reaction (Takara Bio, Japan) and purification with engineered N-terminal Strep-II Tags (Schmidt \& Skerra, 2007).

The coding sequence for $C$. efficiens FAS I (UniProt accession code Q8FMV7) was amplified by PCR from
Table 1

Macromolecule-production information.

\begin{tabular}{ll}
\hline Source organism & C. efficiens \\
DNA source & Genomic DNA \\
Forward primer & CGA AAA AGG CGT CGA CGT GAC CGA ACC \\
& AGG CAG CAA CTT CGG G \\
Reverse primer & GGT GGT GGT GCT CGA GTT AGC CTT CGT \\
& AAC CGG TCG GCT TGA GG \\
Expression vector & pET-22b(+) \\
Expression host & E. coli \\
$\begin{array}{c}\text { Complete amino-acid sequence } \\
\text { of the construct produced }\end{array}$ & UniProtKB Q8FMV7 \\
\hline
\end{tabular}

C. efficiens genomic DNA (DSM 44549). The gene was inserted into an SalI/XhoI-digested pME164 plasmid with an in-fusion cloning reaction (Takara Bio, Japan) to yield pME300 [pME164 is a pET-22b(+) (Merck, Germany) derivative with a N-terminal fused Strep-II Tag-encoding sequence]. Plasmid pME300 was modified using site-directed mutagenesis (forward primer GCG GCG CGC CGC AAC CAG CTG C; reverse primer GGT TGC GGC GCG CCG CGA CAC CCT CCA CGA GTG TC) to generate plasmid pME301 with S1778A and S1779A mutated ACP. Mutations

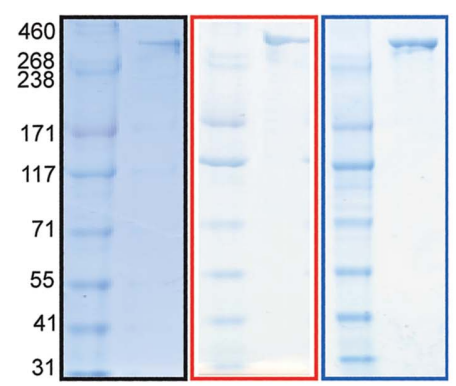

(a)
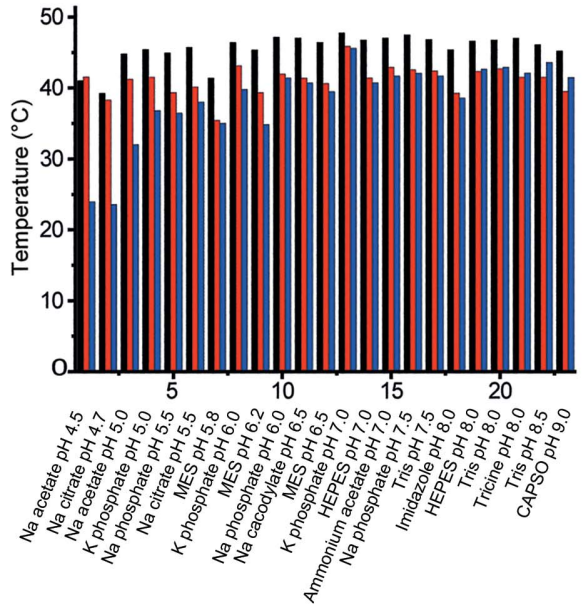

(d)

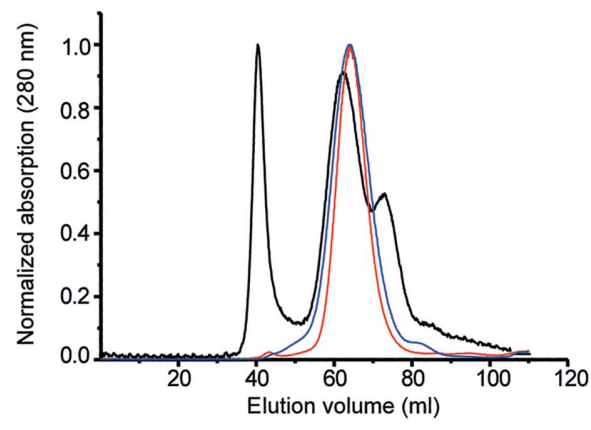

(b)

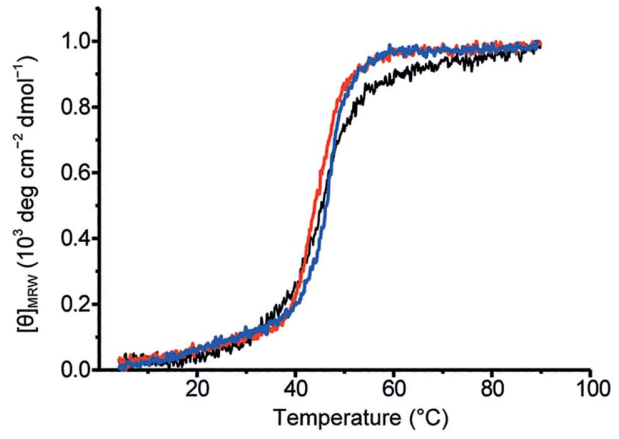

(e)

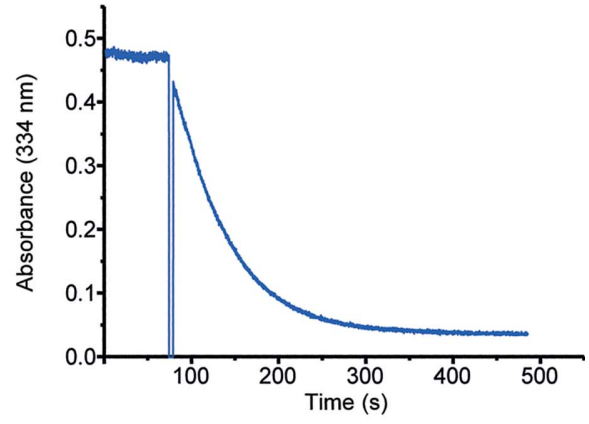

(c)

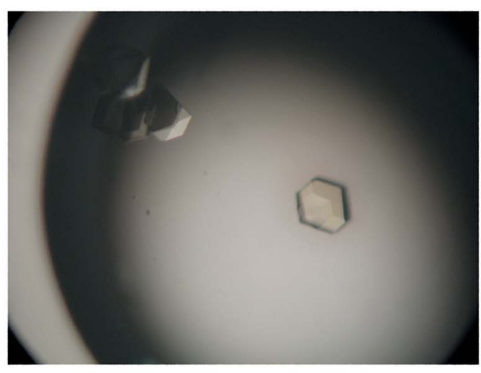

$(f)$

Figure 2

(a) Coomassie-stained SDS gel of purified proteins. Marker is shown in the left lanes (HiMark Protein marker; Life Technologies, USA; labelled in kDa). (b) Superposed size-exclusion chromatograms of proteins, normalized to 1 for the highest peak. The corynebacterial proteins run as monodisperse hexamers, while the M. tuberculosis FAS I chromatographic profile suggests conformational heterogeneity by additional aggregated and dimeric species. Slight shifts in elution volumes might result from different buffer conditions. Buffer conditions: M. tuberculosis FAS I, $0.1 M$ sodium phosphate pH 7.2, $0.1 M \mathrm{NaCl}, 5 \mathrm{~m} M$ sodium malonate; $C$. ammoniagenes, $0.1 M$ sodium phosphate $\mathrm{pH}$ 6.5, $0.1 M \mathrm{NaCl}, 5 \mathrm{~m} M$ sodium malonate; $C$. efficiens, $0.1 M$ bis-tris propane pH 6.8, $0.2 \mathrm{M} \mathrm{NaCl}, 10 \%$ glycerol. (c) Enzymatic activity of $C$. efficiens FAS I monitored by the consumption of NADPH at 334 nm. (d) Fluorescence-based thermal shift assay (Thermofluor assay) for buffer screening. (e) Thermal denaturation of protein monitored by CD spectroscopy. The melting points of the proteins were determined to be $45.4^{\circ} \mathrm{C}$ for $M$. tuberculosis FAS I, $44.6^{\circ} \mathrm{C}$ for C. ammoniagenes FAS I and $47.3^{\circ} \mathrm{C}$ for $C$. efficiens FAS I. $(f)$ Crystals of $C$. efficiens FAS I. Colour code of figure: M. tuberculosis FAS I, black; C. ammoniagenes FAS I, red; $C$. efficiens FAS I, blue. 
were introduced to produce a highly homogeneous batch of non-post-translationally modified protein.

For the cloning of AcpS from C. efficiens in pET-coco-I, the coding sequence was prepared via PCR from genomic DNA (DSM 44549; forward primer AGA AGG AGA TAT AAG CAT GAT CTC GAT TGG AAC CGA TC; reverse primer TCG AGT GCG GCC TAG G CTA CCT GTT CTC GGT GGC CAC). The pET-coco-I plasmid was digested with SphI/ AvrII and assembly was carried out with an in-fusion reaction to yield pME150.

Proteins were purified essentially as described elsewhere (Ciccarelli et al., 2013). Escherichia coli plasmids pME300 and pME301 were transformed into BL21 Gold (DE3) cells (Agilent Technologies, USA). $35 \mathrm{ml} \mathrm{LB}$ medium precultures were inoculated with single ampicillin-resistant colonies and, after overnight growth, were used to further inoculate $21 \mathrm{~TB}$ medium cultures. 21 cultures were grown to an $\mathrm{OD}_{600}$ of 0.8 1.0 at $37^{\circ} \mathrm{C}$ and $180 \mathrm{rev} \mathrm{min}{ }^{-1}$ and then cooled to $20^{\circ} \mathrm{C}$ and induced with a final concentration of $0.5 \mathrm{~m} M$ IPTG. After $16 \mathrm{~h}$, the cells were harvested by centrifugation, frozen in liquid nitrogen and stored at $-80^{\circ} \mathrm{C}$. For purification, cells were resuspended in buffer $W(0.1 M$ sodium phosphate $\mathrm{pH}$ 7.2, $0.15 M \mathrm{NaCl}, 1 \mathrm{~m} M$ EDTA); protease inhibitor (Roche, Switzerland) and DNaseI (Applichem, Germany) were added before breaking the cells with a French press. Lysates were centrifuged for $1 \mathrm{~h}$ at $4^{\circ} \mathrm{C}$ at $45000 \mathrm{~g}$. Supernatants were transferred onto a $10 \mathrm{ml}$ affinity Strep-Tactin gravity-flow column (IBA, Germany), washed five times with one column volume of buffer $W$ and eluted with $6 \times 0.5$ column volumes of buffer $E(0.1 M$ sodium phosphate $\mathrm{pH} 7.2,150 \mathrm{mM} \mathrm{NaCl}$, $1 \mathrm{~m} M$ EDTA, $2.5 \mathrm{~m} M$ desthiobiotin). Proteins were polished by size-exclusion chromatography (SEC) using an ÄKTAexplorer (GE Healthcare, USA) on a Superose 6 XK 16/70 column (GE Healthcare, USA) with buffer $C$ ( $0.1 M$ bis-tris propane $\mathrm{pH} 6.8,0.2 \mathrm{M} \mathrm{NaCl}, 10 \%$ glycerol). Macromoleculeproduction information is summarized in Table 1.

For the preparation of enzymatically active protein, C. efficiens FAS I was co-expressed with C. efficiens AcpS protein. Expression was essentially performed as described above, except that both plasmids pME300 and pME150 were transformed and positive clones were selected by ampicillin/ chloramphenicol double resistance.

Protein stabilities were judged by circular-dichroism (CD) melting curves on a spectropolarimeter (J-715, Jasco, Japan) in buffer $S$ (0.2 $M$ sodium phosphate $\mathrm{pH}$ 7.2). Protein thermal denaturation by the Thermofluor method was carried out in a LightCycler 480 (Roche, Mannheim, Germany) and visualized using SYPRO Orange (Life Technologies, USA; Fig. 2d; Ericsson et al., 2006).

Preparations of $C$. efficiens FAS I from co-expression with AcpS were tested for FAS activity in a NADPH consumption assay (Lynen, 1969). For the assay, in a total volume of $100 \mu \mathrm{l}$, $25 \mu \mathrm{g}$ FAS I in buffer $A B(0.4 M$ potassium phosphate $\mathrm{pH}$ 7.3, $3 \mathrm{~m} M$ dithiothreitol) was incubated with $30 \mathrm{n} M$ NADPH (Sigma-Aldrich, USA) and $50 \mathrm{n} M$ acetyl-CoA (SigmaAldrich, USA). The absorption was recorded at $334 \mathrm{~nm}$ (Lambda 35, PerkinElmer, USA) for approximately $1 \mathrm{~min}$
Table 2

Crystallization.

\begin{tabular}{|c|c|}
\hline Method & Vapour diffusion \\
\hline Plate type & $\begin{array}{l}\text { Greiner CrystalQuick plate (screening), } \\
\text { Crystalgen SuperClear 24-well plates } \\
\text { (final) }\end{array}$ \\
\hline Temperature (K) & 277 and 293 \\
\hline ration $\left(\mathrm{mg} \mathrm{ml}^{-1}\right)$ & 10 \\
\hline $\begin{array}{l}\text { Buffer composition of protein } \\
\text { solution }\end{array}$ & $\begin{array}{l}0.1 M \text { bis-tris propane } \mathrm{pH} 6.8,0.15 M \\
\mathrm{NaCl}, 10 \% \text { glycerol }\end{array}$ \\
\hline $\begin{array}{l}\text { Composition of reservoir } \\
\text { solution }\end{array}$ & $\begin{array}{l}0.1-0.3 M \text { sodium malonate, } 0.1 M \text { bis-tris } \\
\text { propane pH } 7.5,17-22 \% \text { PEG } 3350\end{array}$ \\
\hline Volume and ratio of drop & $1: 1$ ratio protein:reservoir, $2 \mu \mathrm{l}$ final volum \\
\hline Volume of reservoir $(\mu \mathrm{l})$ & 100 (screening), 600 (final) \\
\hline
\end{tabular}

before $60 \mathrm{n} M$ malonyl-CoA (Sigma-Aldrich, USA) was added to the solution (Fig. 2c).

For determination of the product spectra, reaction solutions with the above-described composition were incubated overnight at room temperature. After $16 \mathrm{~h}, \mathrm{C}_{17}-\mathrm{CoA}$ (SigmaAldrich, USA) was added as an internal standard and the reactions were stopped by adding precooled $\left(-20^{\circ} \mathrm{C}\right)$ acetone. These solutions were mixed for $20 \mathrm{~s}$ and stored for $60 \mathrm{~min}$ at $-20^{\circ} \mathrm{C}$. After centrifugation for $5 \mathrm{~min}$ at $20000 \mathrm{~g}$, the supernatants were evaporated in a SpeedVac at $4^{\circ} \mathrm{C}$. The CoA esters were dissolved in $60 \mu \mathrm{l}$ Milli-Q water and, after ultrasonication, were analysed by HPLC-UV-MS [Ultimate 3000 RSLC (Thermo Fisher Scientific, USA) coupled to a micrOTOF-Q II system (Bruker Daltonics, Germany)]. CoA esters were separated on an RP-18 column $(100 \times 2.1 \mathrm{~mm}$, particle size $1.7 \mu \mathrm{m}$; Waters, USA) in gradients of solvent $A$ (water, $10 \mathrm{~m} M$ triethylamine/acetic acid buffer $\mathrm{pH} 9.0$ ) and $B$ (acetonitrile). Data were analyzed using the DataAnalysis 4.0 software package (Bruker Daltonics, Germany).

\subsection{Crystallization}

Despite extensive crystallization trials, both M. tuberculosis and $C$. ammoniagenes FAS I gave crystals that diffracted to only about $8 \AA$ resolution (data not shown). Hence, we chose another sequence homologue, FAS I from C. efficiens, for its reported moderate thermotolerance and the expected higher conformational stability of its proteins (Nishio et al., 2003). Initial crystallization conditions for C. efficiens FAS I were identified by carrying out screening trials using various commercial sparse-matrix crystallization screening kits (The AmSO4 Suite, The Cations Suite, The JCSG+ Suite, The JCSG Core I-IV Suites, The PACT Suite, The PEGs Suite and The PEGs II Suite from Qiagen, Index HT from Hampton Research, and Morpheus HT-96 and The PGA Screen from Molecular Dimensions) using the sitting-drop vapourdiffusion method in 96-well Greiner plates at 4 and $22^{\circ} \mathrm{C}$. Tiny crystals were observed in The PACT Suite at $4^{\circ} \mathrm{C}$ after $2 \mathrm{~d}$. This condition was refined by the hanging-drop vapourdiffusion technique in 24-well plates using $1 \mu \mathrm{l}$ protein solution in the droplet mixed with $1 \mu \mathrm{l}$ reservoir solution. The best crystals were obtained at $0.1-0.3 \mathrm{M}$ sodium malonate, $0.1 \mathrm{M}$ bis-tris propane $\mathrm{pH} 7.5,17-22 \%$ PEG 3350 at $4^{\circ} \mathrm{C}$. Crystals grew after about one week with sizes ranging from $100 \times 100$ $\times 75$ to $150 \times 150 \times 75 \mu \mathrm{m}$ (Fig. $2 f$ ). Crystallization 
information is summarized in Table 2. C. efficiens FAS I crystals were soaked with $\mathrm{Ta}_{6} \mathrm{Br}_{12}$ (Jena Bioscience), $\mathrm{W}_{12}$ (Alfa Aesar), $\mathrm{W}_{18}$ (a gift from Professor Robert Huber) or Nanogold (Nanoprobes) for $10 \mathrm{~min}, 1 \mathrm{~h}, 2 \mathrm{~h}$ or overnight. The concentrations of the soaking solutions for all clusters were varied in the low-micromolar range. For co-crystallization, the heavy atoms were added to the abovementioned crystallization conditions to a final concentration that was in the lowmicromolar range. In both cases, the crystals showed poor diffraction ( $7 \AA$ or worse) when exposed to X-rays and rapid decay in diffraction intensities after a few exposures. Data sets could not be collected.

\subsection{Data collection and processing}

C. efficiens FAS I crystals were transferred to a cryoprotectant solution consisting of $20 \%$ ethylene glycol in the crystallization buffer, picked up in a nylon-fibre loop and plunged into liquid nitrogen. All crystals were exposed to single-wavelength X-radiation on beamline ID14-4 at the European Synchrotron Radiation Facility (ESRF) and maintained at $100 \mathrm{~K}$ while data were recorded on a CCD detector (ADSC Quantum Q315r). Individual diffraction images from C. efficiens FAS I crystals were initially indexed with iMosflm (Battye et al., 2011) to determine the crystal form. Data were processed with the $x i a 2$ suite of programs (Winter, 2010) running the XDS package (Kabsch, 2010) and AIMLESS (Evans, 2006). Crystals gave two different crystal forms in space groups $R 32$ and $C 2$ with differing unit-cell parameters (Table 3), designated crystal forms I and II. All diffraction data are publicly available at Zenodo (http://dx.doi.org/ 10.5281/zenodo.20031).

\section{Results and discussion}

We have recently established protocols for the recombinant overexpression of $M$. tuberculosis and C. ammoniagenes FAS I. C. efficiens, which is evolutionarily related to C. ammoniagenes, is reported to be moderately thermotolerant (Nishio et al., 2003), and we expected a higher conformational stability of its protein inventory. Two FAS I-coding genes (FAS A, NP_737523.1, and FAS B, NP_739002.1) were identified in C. efficiens (Stuible et al., 1997). FAS B (UniProt accession code Q8FMV7) was picked as a target for our studies owing to its higher sequence identity to M. tuberculosis FAS I (44 and $52 \%$ for FAS A and FAS B, respectively). About $5 \mathrm{mg}$ of protein per litre of E. coli expression culture was obtained after chromatographic purification (Fig. 2a). The hexameric conformation was monitored by size-exclusion chromatography (Fig. $2 b$ ), and catalytic activity was demonstrated by spectroscopically monitoring NADPH consumption during the reductive steps in fatty-acid synthesis. The specific activity was determined as $270 \pm 55 \mathrm{mU} \mathrm{mg}^{-1}$, which is comparable to previously reported values (Fig. 2c; Stuible et al., 1997). Under the assay conditions, C. efficiens FAS I produced $\mathrm{C}_{16}$-CoA (86\%) and $\mathrm{C}_{18}$-CoA $(14 \%)$. The protein stability of C. efficiens FAS I, as well as the homologous FAS I from C. ammonia-
Table 3

Data collection and processing.

Values in parentheses are for the outer shell.

\begin{tabular}{|c|c|c|}
\hline & Crystal form 1 & Crystal form 2 \\
\hline Diffraction source & ID14-4, ESRF & ID14-4, ESRF \\
\hline Wavelength $(\AA)$ & 0.939 & 0.939 \\
\hline Temperature $(\mathrm{K})$ & 100 & 100 \\
\hline Detector & $\begin{array}{l}\text { ADSC Quantum } \\
\text { Q315r }\end{array}$ & $\begin{array}{l}\text { ADSC Quantum } \\
\text { Q315r }\end{array}$ \\
\hline Crystal-to-detector distance $(\mathrm{mm})$ & 628 & 680 \\
\hline Rotation range per image $\left(^{\circ}\right)$ & 1 & 0.2 \\
\hline Total rotation range $\left({ }^{\circ}\right)$ & 407 & 180 \\
\hline Exposure time per image (s) & 1.2 & 0.3 \\
\hline Space group & $R 32$ & $C 2$ \\
\hline$a, b, c(\AA)$ & $337.9,337.9,246.7$ & $243.9,330.4,214.1$ \\
\hline$\alpha, \beta, \gamma\left({ }^{\circ}\right)$ & $90,90,120$ & $90,115,90$ \\
\hline Mosaicity $\left(^{\circ}\right)$ & 0.3 & 0.1 \\
\hline Resolution range $(\AA)$ & $50-6.0(7.6-6.0)$ & $20-4.5(4.6-4.5)$ \\
\hline Total No. of reflections & 338393 & 339618 \\
\hline No. of unique reflections & 13665 & 90165 \\
\hline Completeness (\%) & $100(100)$ & $100(100)$ \\
\hline Multiplicity & $24(25)$ & $3.8(3.8)$ \\
\hline$\langle I / \sigma(I)\rangle$ & $12(1) \dagger$ & $5.2(1.4) \ddagger$ \\
\hline$R_{\text {r.i.m. }}$ & $0.22(4.2)$ & $0.21(1.1)$ \\
\hline $\begin{array}{l}\text { Overall } B \text { factor from Wilson } \\
\text { plot }\left(\AA^{2}\right)\end{array}$ & 350 & 162 \\
\hline
\end{tabular}

† Data were scaled to a $\mathrm{CC}_{1 / 2}$ (Karplus \& Diederichs, 2012) of 0.5. The mean $I / \sigma(I)$ falls below 2.0 at $6.5 \AA$ resolution. \# Data were scaled to a $\mathrm{CC}_{1 / 2}$ (Karplus \& Diederichs, 2012) of 0.5 . The mean $I / \sigma(I)$ falls below 2.0 at $4.7 \AA$ resolution.

genes and M. tuberculosis, was analyzed by the fluorescencebased thermal shift (Thermofluor) assay and CD spectroscopy. Melting points of $45.4^{\circ} \mathrm{C}$ for $M$. tuberculosis FAS I, $44.6^{\circ} \mathrm{C}$ for C. ammoniagenes FAS I and $47.3^{\circ} \mathrm{C}$ for $C$. efficiens FAS I were determined, indicating low thermal stabilities of bacterial FAS I (Figs. $2 d$ and $2 e$; Ericsson $e t$ al., 2006). Aiming for the highest conformational homogeneity, the structure of $C$. efficiens FAS I was characterized on a mutant construct bearing the modifications S1178A and S1779A. These mutations prevent posttranslational phosphopantetheinylation of the protein and should avoid the binding of intermediates or substrates to ACP that could induce different conformational states (Whicher et al., 2014). This was carried out despite the observation that $C$. efficiens FAS I is inactive when expressed alone without $C$. efficiens AcpS.

Despite several optimization trials (in terms of protein purification, crystallization and post-crystallization protocols; e.g. the use of dehydration), diffraction data from the crystals could be acquired to only $4.5 \AA$ resolution. Data sets from two crystals gave different unit-cell parameters in space groups $R 32$ and $C 2$, referred to as crystal forms I and II, respectively (Table 3). The packing of the FAS molecule in the asymmetric unit of the crystal was analyzed using MATTHEWS_COEF (Matthews, 1968) from the CCP4 suite (Winn et al., 2011). For crystal form I, calculation of the Matthews coefficient $\left(V_{\mathrm{M}}\right)$ gave values of 4,2 and $1.3 \AA^{3} \mathrm{Da}^{-1}$, corresponding to solvent contents of 69,38 and $7.1 \%$ for one, two and three molecules per asymmetric unit, respectively. This indicated the presence of one molecule in the asymmetric unit and the absence of noncrystallographic symmetry. In case of crystal form II, up to six chains (a whole hexamer barrel) can be accommodated in the asymmetric unit, indicating clear noncrystallographic 
symmetry. For crystal form II, search templates comprising the hexameric barrel, a trimeric dome, a dimer or a monomer individually did not yield unique solutions in the molecularreplacement analysis.

In the case of crystal form I, the phase problem could be solved by molecular replacement (MR) using the structural model of M. smegmatis FAS I (Boehringer et al., 2013; PDB entry 3zen) as a model in MOLREP (Vagin \& Teplyakov, 2010 ) with the help of the CCP4 suite. The MR solution was confirmed with Phaser (McCoy et al., 2007), which gave an LLG score of 424 and a TFZ score of 26. It should be noted that the overall sequence identity between $C$. efficiens FAS I and M. smegmatis FAS I is $53 \%$ (with $67 \%$ positives and $4 \%$ gaps; data from BLAST; Altschul et al., 1990). A pairwise alignment between the protein sequences of $M$. smegmatis FAS I and C. efficiens FAS I was used in phenix.sculptor (Bunkóczi \& Read, 2011) from the PHENIX package (Adams et al., 2010) to mutate the MR solution (PDB) file to the C. efficiens FAS I sequence. An immediate rigid-body refinement with the different domains (sequence ranges: AT, 10367; ER, 368-879; SBS, 880-1002; DH, 1003-1289; MAT, 12901674; DM, 1953-2065; KR, 2073-2357; KS, 2358-3022) as independent rigid groups using PHENIX gave $R$ and $R_{\text {free }}$ values of 0.32 and 0.40 , respectively. Although the diffraction data set is of low resolution, a preliminary view of the electron-density map displayed the different domains of this megadalton molecule. In the next step, we attempted model improvement using the phenix.morph_model command (Terwilliger et al., 2012) invoking the prime-and-switch map (Terwilliger, 2004), but did not observe any significant improvement in either the refinement $\left(R / R_{\text {free }}\right)$ values or the electron density.

\section{Acknowledgements}

We are grateful to Dieter Oesterhelt, who supported this project in its early phase. We are also grateful to Werner Kühlbrandt for his continuous support of our research focus on the structural characterization of FAS megaenzymes. We thank Jan Gajewski for cloning C. efficiens AcpS and for collecting LC-MS data on the C. efficiens FAS I product spectrum. We also thank Alexander Rittner and David Wirthensohn for assistance when starting this project and for valuable discussions. Lastly, we thank Andreas Bracher for his assistance in synchrotron data-collection trips and the EMBLESRF Joint Structural Biology Group for help on the ESRF beamlines. This work was supported by the German Research Foundation (DFG Project GR3854 to MG) and by the Cluster of Excellence Frankfurt (CEF) 'Macromolecular Complexes' at the Goethe University Frankfurt (CEF Adjunct Investigatorship Program). Finally, MG is grateful for funding from the Volkswagen Foundation (Lichtenberg Professorship).

\section{References}

Adams, P. D. et al. (2010). Acta Cryst. D66, 213-221.

Altschul, S. F., Gish, W., Miller, W., Myers, E. W. \& Lipman, D. J. (1990). J. Mol. Biol. 215, 403-410.
Banerjee, A., Dubnau, E., Quemard, A., Balasubramanian, V., Um, K. S., Wilson, T., Collins, D., de Lisle, G. \& Jacobs, W. R. Jr (1994). Science, 263, 227-230.

Battye, T. G. G., Kontogiannis, L., Johnson, O., Powell, H. R. \& Leslie, A. G. W. (2011). Acta Cryst. D67, 271-281.

Bhatt, A., Molle, V., Besra, G. S., Jacobs, W. R. Jr \& Kremer, L. (2007). Mol. Microbiol. 64, 1442-1454.

Boehringer, D., Ban, N. \& Leibundgut, M. (2013). J. Mol. Biol. 425, 841-849.

Brignole, E. J., Smith, S. \& Asturias, F. J. (2009). Nature Struct. Mol. Biol. 16, 190-197.

Bunkóczi, G. \& Read, R. J. (2011). Acta Cryst. D67, 303-312.

Ciccarelli, L., Connell, S. R., Enderle, M., Mills, D. J., Vonck, J. \& Grininger, M. (2013). Structure, 21, 1251-1257.

Ericsson, U. B., Hallberg, B. M., DeTitta, G. T., Dekker, N. \& Nordlund, P. (2006). Anal. Biochem. 357, 289-298.

Evans, P. (2006). Acta Cryst. D62, 72-82.

Gago, G., Diacovich, L., Arabolaza, A., Tsai, S.-C. \& Gramajo, H. (2011). FEMS Microbiol. Rev. 35, 475-497.

Gannoun-Zaki, L., Alibaud, L. \& Kremer, L. (2013). Antimicrob. Agents Chemother. 57, 629-632.

Gipson, P., Mills, D. J., Wouts, R., Grininger, M., Vonck, J. \& Kuhlbrandt, W. (2010). Proc. Natl Acad. Sci. USA, 107, 9164-9169.

Grininger, M. (2014). Curr. Opin. Struct. Biol. 25, 49-56.

Jenni, S., Leibundgut, M., Boehringer, D., Frick, C., Mikolasek, B. \& Ban, N. (2007). Science, 316, 254-261.

Johansson, P., Mulinacci, B., Koestler, C., Vollrath, R., Oesterhelt, D. \& Grininger, M. (2009). Structure, 17, 1063-1074.

Johansson, P., Wiltschi, B., Kumari, P., Kessler, B., Vonrhein, C., Vonck, J., Oesterhelt, D. \& Grininger, M. (2008). Proc. Natl Acad. Sci. USA, 105, 12803-12808.

Kabsch, W. (2010). Acta Cryst. D66, 125-132.

Karplus, P. A. \& Diederichs, K. (2012). Science, 336, 1030-1033.

Kikuchi, S., Rainwater, D. L. \& Kolattukudy, P. E. (1992). Arch. Biochem. Biophys. 295, 318-326.

Kremer, L., Douglas, J. D., Baulard, A. R., Morehouse, C., Guy, M. R., Alland, D., Dover, L. G., Lakey, J. H., Jacobs, W. R. Jr, Brennan, P. J., Minnikin, D. E. \& Besra, G. S. (2000). J. Biol. Chem. 275, 16857-16864.

Kresze, G.-B., Streber, L., Oesterhelt, D. \& Lynen, F. (1977). Eur. J. Biochem. 79, 191-199.

Leibundgut, M., Jenni, S., Frick, C. \& Ban, N. (2007). Science, 316, 288-290.

Lomakin, I. B., Xiong, Y. \& Steitz, T. A. (2007). Cell, 129, 319-332.

Lynen, F. (1969). Methods Enzymol. 14, 17-33.

Ma, Z., Lienhardt, C., McIlleron, H., Nunn, A. J. \& Wang, X. (2010). Lancet, 375, 2100-2109.

Maier, T., Leibundgut, M., Boehringer, D. \& Ban, N. (2010). Q. Rev. Biophys. 43, 373-422.

Matthews, B. W. (1968). J. Mol. Biol. 33, 491-497.

McCoy, A. J., Grosse-Kunstleve, R. W., Adams, P. D., Winn, M. D., Storoni, L. C. \& Read, R. J. (2007). J. Appl. Cryst. 40, 658-674.

Nishio, Y., Nakamura, Y., Kawarabayasi, Y., Usuda, Y., Kimura, E., Sugimoto, S., Matsui, K., Yamagishi, A., Kikuchi, H., Ikeo, K. \& Gojobori, T. (2003). Genome Res. 13, 1572-1579.

Okonechnikov, K., Golosova, O. \& Fursov, M. (2012). Bioinformatics, 28, 1166-1167.

Peterson, D. O. \& Bloch, K. (1977). J. Biol. Chem. 252, 5735-5739.

Sacco, E., Covarrubias, A. S., O'Hare, H. M., Carroll, P., Eynard, N., Jones, T. A., Parish, T., Daffé, M., Bäckbro, K. \& Quémard, A. (2007). Proc. Natl Acad. Sci. USA, 104, 14628-14633.

Sayahi, H., Zimhony, O., Jacobs, W. R. Jr, Shekhtman, A. \& Welch, J. T. (2011). Bioorg. Med. Chem. Lett. 21, 4804-4807.

Schmidt, T. G. \& Skerra, A. (2007). Nature Protoc. 2, 1528-1535.

Schweizer, E. \& Hofmann, J. (2004). Microbiol. Mol. Biol. Rev. 68, 501-517.

Stuible, H.-P., Meurer, G. \& Schweizer, E. (1997). Eur. J. Biochem. 247, 268-273. 
Sullivan, T. J., Truglio, J. J., Boyne, M. E., Novichenok, P., Zhang, X., Stratton, C. F., Li, H.-J., Kaur, T., Amin, A., Johnson, F., Slayden, R. A., Kisker, C. \& Tonge, P. J. (2006). ACS Chem. Biol. 1, 43-53. Sumper, M., Oesterhelt, D., Riepertinger, C. \& Lynen, F. (1969). Eur. J. Biochem. 10, 377-387.

Terwilliger, T. C. (2004). Acta Cryst. D60, 2144-2149.

Terwilliger, T. C., Read, R. J., Adams, P. D., Brunger, A. T., Afonine, P. V., Grosse-Kunstleve, R. W. \& Hung, L.-W. (2012). Acta Cryst. D68, 861-870.

Vagin, A. \& Teplyakov, A. (2010). Acta Cryst. D66, 22-25.
Whicher, J. R., Dutta, S., Hansen, D. A., Hale, W. A., Chemler, J. A., Dosey, A. M., Narayan, A. R., Håkansson, K., Sherman, D. H., Smith, J. L. \& Skiniotis, G. (2014). Nature (London), 510, 560564.

Winn, M. D. et al. (2011). Acta Cryst. D67, 235-242.

Winter, G. (2010). J. Appl. Cryst. 43, 186-190.

Zimhony, O., Cox, J. S., Welch, J. T., Vilchèze, C. \& Jacobs, W. R. Jr (2000). Nature Med. 6, 1043-1047.

Zimhony, O., Vilchèze, C. \& Jacobs, W. R. Jr (2004). J. Bacteriol. 186, 4051-4055. 